\title{
MODELO DE DESIGN PARA O COMPORTAMENTO SAUDÁVEL COMO SUPORTE PARA PROJETOS DE EDUCAÇÃO NUTRICIONAL INFANTIL.
}

\section{THE DESIGN MODEL FOR HEALTHY BEHAVIOR AS SUPPORT FOR CHILD NUTRITION EDUCATION PROJECTS.}

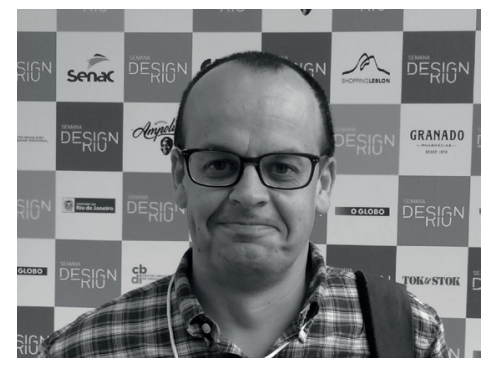

Filipe Campelo Xavier da Costa

Doutor em Administração

Programa de Pós-graduação em Design

(Universidade do Vale do Rio dos Sinos - UNISINOS)

e-mail: fcampelo@unisinos.br

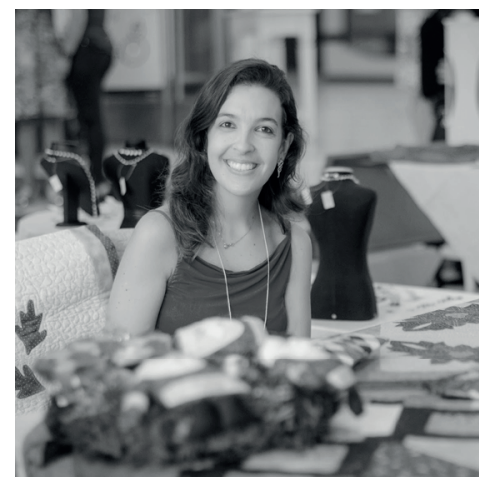

\section{Juliana Gonçalves Mota}

Mestre em Design

Programa de Pós-graduação em Design

(Universidade do Vale do Rio dos Sinos - UNISINOS)

e-mail: julianagmota@gmail.com 


\section{RESUMO}

Este artigo aborda o processo de aplicação do Modelo de Design para o Comportamento Saudável (MDCS - Ludden e Hekkert, 2014) como suporte para a criação de jogos focados no estímulo da alimentação saudável infantil. O MDCS foi desenvolvido para potencializar projetos que visam incentivar atitudes para o bem-estar dos usuários. Utilizando-se os princípios do MDCS na condução do processo de projeto, foi realizado um workshop com a participação de designers e mães com filhos em idade pré-escolar. $O$ workshop desafiou os participantes a criar jogos de interação entre as crianças e os alimentos saudáveis. A partir da análise da dinâmica criativa das equipes, este artigo discute as características e potencialidades do MDCS como um instrumento para aperfeiçoar os processos projetuais focados na alimentação saudável infantil e prospectar novas possibilidades de projeto para prevenção da obesidade.

\section{PALAVRAS-CHAVE}

Modelo de Design para o Comportamento Saudável; Processo de Projeto; Nutrição Infantil.

\section{ABSTRACT}

This paper addresses the application process of the Design for Healhty Behavior Framework (DHBF) as support for the creation of games focused on stimulating healthy infant feeding. The DHBF was developed to potentiate projects that aim to strengthen attitudes towards the well-being of users. Using the DHBF principles in conducting design process, a workshop was held with the participation of designers and mothers of pre-school children. The workshop challenged the participants to create interaction games between children and healthy food. From the analysis of the creative dynamics of the teams, this paper discusses the characteristics and potentialities of the DHBF as an instrument to improve the design processes focused on healthy child nutrition and prospect new project possibilities to obesity prevention.

\section{KEYWORDS}

Design for Healthy Behavior Framework; Design Process; Child Nutrition.

\section{INTRODUÇÃO}

O papel do design, na perspectiva de Buchanan (2001), extrapola a criação de produtos, passando a interferir sobre o comportamento dos usuários e sobre 
a forma "[...] como os seres humanos relacionam-se através da influência mediadora dos produtos" (p. 11, tradução nossa). Convergindo com esta ideia, Bhamra, Lilley e Tang (2008) afirmam que é possível moldar o comportamento dos usuários por meio do design, pois criar artefatos "significa projetar para a experiência do usuário com o produto, o que também determina a combinação de impactos desta experiência" (p. 2, tradução nossa). Neste contexto, o design constitui-se como um importante condutor de transformação e inovação, uma vez que possui conhecimentos e habilidades que possibilitam "descobrir do que as pessoas necessitam, o que desejam, como elas interpretam os produtos que utilizam e como isso afeta as suas experiências" (TROMP, 2013, p. 10, tradução nossa).

Para criar artefatos com maiores chances de influenciar o comportamento dos usuários, designers e pesquisadores identificaram a necessidade de aprofundar seus conhecimentos a respeito da formação dos hábitos e escolhas dos indivíduos. Tal condição motivou a aproximação do design a preceitos teóricos da psicologia, proporcionando a emergência de um amplo espectro de abordagens reconhecido como design para mudança de comportamento. O princípio deste campo do design é transformar a compreensão do comportamento humano em estratégias para a criação de soluções que impactem positivamente a vida dos indivíduos e a sociedade (LOCKTON; HARRISON; STANTON, 2010).

Uma das abordagens que se destaca entre as pesquisas para mudança de comportamento é o design para o bem-estar. Esta área abrange diferentes métodos de projeto com o intuito de engajar o usuário em hábitos saudáveis e de criar soluções para a prevenção de enfermidades como a depressão, o diabetes e a obesidade (NIEDDERER et al., 2014). Para atender a este fim, foi desenvolvido o Modelo de Design para o Comportamento Saudável (MDCS - LUDDEN; HEKKERT, 2014). O modelo associa as etapas do projeto aos estágios da mudança comportamental, sugerindo diferentes estratégias de design para promover o processo de aprendizagem de hábitos saudáveis.

No âmbito da nutrição infantil, contexto de aplicação deste estudo, os prognósticos sobre a obesidade motivaram a criação de diversas campanhas para incentivar a alimentação saudável entre as crianças. O crescimento contínuo destes índices, no entanto, sugere que os atuais meios para lidar com o problema, embora relevantes, resultam em um impacto mínimo sobre o comportamento nutricional infantil (MURILLO et al., 2016). Esta constatação indica como uma alternativa para o problema o desenvolvimento de projetos que visem estimular hábitos alimentares saudáveis na infância.

Diante do potencial do design para mudança de comportamento em criar ar- 
tefatos que ajudam a gerar impactos sociais positivos, este artigo analisa o processo de aplicação do MDCS como suporte para a criação de jogos focados no estímulo de hábitos alimentares saudáveis na infância. Para tanto, realizou-se em setembro de 2016 um workshop no qual foi possível estabelecer a interação colaborativa entre designers e usuárias (mães), no papel de principais educadoras nutricionais dos filhos. A partir da análise do processo projetual das equipes, este artigo discute as características e potencialidades do MDCS como um instrumento para potencializar projetos orientados à mudança comportamental dos usuários e à prevenção da obesidade infantil.

\section{MODELO DE DESIGN PARA O COMPORTAMENTO SAUDÁVEL}

Além de encorajar comportamentos sustentáveis - como o Modelo para o Comportamento Sustentável proposto por Lilley (2009) - e atitudes para a prevenção de crimes $^{1}$, o campo do design para mudança de comportamento compreende abordagens focadas na promoção do bem-estar dos usuários. Uma das propostas de destaque nesta área é o modelo desenvolvido por Ludden e Hekkert (2014), denominado neste estudo de Modelo de Design para o Comportamento Saudável (MDCS). O objetivo desta proposta é potencializar o desenvolvimento de artefatos que estimulem hábitos para uma vida saudável.

O MDCS foi construído com base no Modelo Transteórico de Mudança Comportamental (PROCHASKA; DICLEMENTE; NORCROSS, 1992). De acordo com esta perspectiva, a construção de hábitos em longo prazo exige que as pessoas perpassem por cinco estágios dentro de uma lógica temporal: pré-contemplação, contemplação, preparação, ação e manutenção ².

O MDCS parte do princípio de que, para criar artefatos que incentivem a mudança de atitude do usuário, os designers devem considerar em qual destes estágios o indivíduo se encontra no processo de mudança comportamental

1 Maiores informações podem ser acessadas em:<http://www.designagainstcrime. com>.

2 No estágio de pré-contemplação, os indivíduos ainda não estão cientes das consequências de suas atitudes atuais e da necessidade da mudança. Portanto, eles não se encontram ainda preparadas para este fim e não têm o propósito de mudar pelos próximos seis meses. $\mathrm{Na}$ fase seguinte (contemplação), manifestam-se as primeiras intenções de transformação, e os benefícios de um novo comportamento tornam-se mais evidentes. A intenção de mudar no curto prazo emerge no estágio de preparação, quando as pessoas já têm em mente um plano de ação. A etapa da ação, por sua vez, compreende o período em que o novo comportamento será realizado no curto prazo. E, por fim, na fase de manutenção, o processo de mudança já está instituído, sustentando-se por um período acima de seis meses (PROCHASKA; VELICER, 1997). 
e o quão motivado ele está para alcançar este objetivo. Desconsiderar este princípio implica a criação de artefatos que não respeitam as reais motivações dos indivíduos, podendo assumir um caráter impositivo e, consequentemente, ter menos chance de serem reconhecidos pelo usuário como um suporte para o seu bem-estar (LUDDEN; HEKKERT, 2014).

Com base neste entendimento, o princípio básico do MDCS é associar diferentes etapas do processo de design aos cinco estágios da mudança do comportamento do usuário apontados anteriormente. Na perspectiva de Ludden e Hekkert (2014), o projeto de artefatos para estimular o comportamento saudável deve conter quatro etapas: sensibilização, capacitação, motivação e atenuação gradual da intervenção (Figura 1).

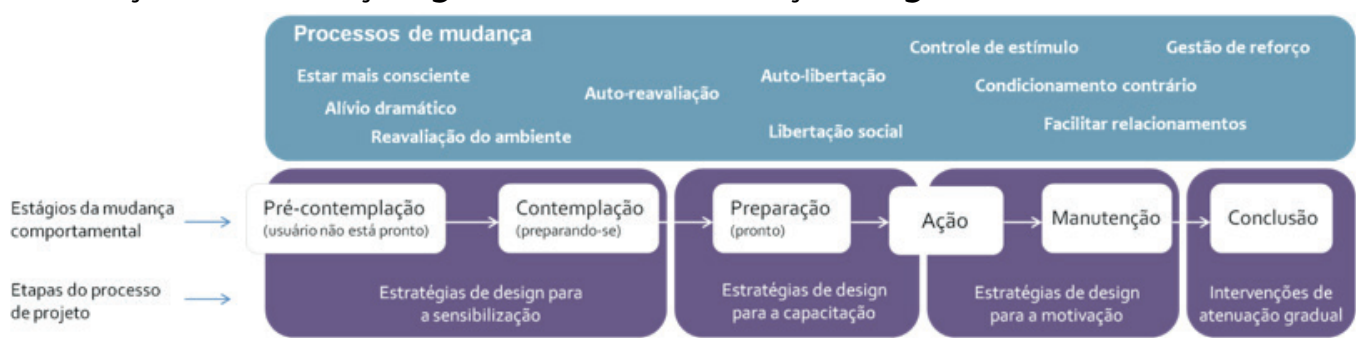

Figura 1: Modelo de Design para o Comportamento Saudável (MDCS). Fonte: adaptada de Ludden e Hekkert (2014, p. 486).

Para cada etapa do processo de projeto, o modelo atribui diferentes estratégias, a fim de que os designers possam projetar artefatos para a mudança de comportamento do usuário com mais eficiência. As estratégias de sensibilização estão voltadas ao indivíduo que não está preparado para a mudança comportamental. Elas têm por objetivo aumentar o seu nível de consciência acerca da importância dos hábitos saudáveis. As estratégias de capacitação atingem o usuário que já conhece os benefícios do novo comportamento, por isso envolvem a criação estímulos para conferir confiança e autonomia para que o indivíduo avance no processo de mudança. As estratégias de motivação atuam sobre o sujeito que já assimilou o comportamento saudável, mas que precisa de incentivos constantes para transformá-lo em hábito. Trata-se de estratégias para o design de artefatos que ajudem o usuário a aprender o novo comportamento e manter as práticas saudáveis. A última fase do modelo, a atenuação gradual, compreende a criação de estímulos para fortalecer o novo hábito, mas de forma a suavizarem-se gradualmente, a fim de que o usuário assuma a autonomia no processo (LUDDEN; HEKKERT, 2014).

Na parte superior do modelo, são apresentadas técnicas de mudança de comportamento, como a autorreavaliação e o alívio dramático, que foram analisa- 
das em estudos clínicos da abordagem teórica da Psicologia conhecida como Modelo Transteórico de Mudança Comportamental (PROCHASKA; DICLEMENTE; NORCROSS, 1992). Estes processos, no entanto, não foram observados neste artigo, uma vez que não estão compreendidos no escopo teórico da pesquisa. Ludden e Hekkert (2014) afirmam que a associação das fases da mudança comportamental ao processo de projeto permite aos designers criar soluções para o jeito com que as pessoas efetivamente se comportam e não para o modo como elas gostariam de agir. Tal associação é fundamental, uma vez que os artefatos para a mudança de comportamento somente serão reconhecidos pelos usuários se eles estiverem combinados com o seu estado de motivação no processo de transformação (LUDDEN; HEKKERT, 2014). Projetado de tal forma, o artefato respeita a disposição interna - ou o estágio motivacional - do usuário e tem mais potencial de assumir um significado para eles. Com base no MDCS, os designers podem projetar com mais eficiência artefatos para auxiliar a transformação comportamental dos usuários.

Com o objetivo de potencializar a criação de soluções para estimular a alimentação saudável entre as crianças, o MDCS foi aplicado em um workshop como um instrumento facilitador do processo de projeto. A escolha por este modelo se justifica, na medida em que as intervenções de design sugeridas por Ludden e Hekkert (2014) ajudam a manter o foco do projeto no bem-estar do usuário, colocando suas necessidades e motivações no centro do processo. Ao associar estratégias de design a conceitos da psicologia para a saúde, o MDCS pode contribuir com uma compreensão mais apurada acerca das necessidades dos usuários e de como se deve projetar artefatos para engajá-los em práticas saudáveis de forma eficaz.

\section{O MDCS COMO SUPORTE NA CRIAÇÃO DE JOGOS PARA A EDUCAÇÃO ALIMENTAR}

O MDCS foi aplicado em um workshop realizado em setembro de 2016, dentro do Programa de Pós-graduação em Design da Unisinos (Porto Alegre - RS). A dinâmica contou com a participação voluntária de seis alunos do sexto semestre do curso de graduação em design da mesma instituição e de 13 mães de crianças em 2 e 6 anos das classes B e C, segundo o Critério de Classificação Econômica Brasil ${ }^{3}$. Formaram-se quatro equipes de trabalho, cujo desafio era criar jogos com atividades de interação positiva entre as crianças e os alimentos saudáveis. No briefing foi solicitado às equipes que desenvolvessem atividades que respon3 Disponível em: http://www.abep.org/criterio-brasil. Acesso em: 13 ago. 2016. 
dessem apenas às três primeiras etapas que compõe o modelo (sensibilização, capacitação e motivação). Como meio de aplicar as etapas do MDCS no workshop e torná-las mais compreensíveis aos participantes, o briefing estabeleceu que as equipes criassem jogos organizados em três fases, desenvolvendo uma atividade de interação para cada etapa correspondente do modelo de design.

Optou-se por considerar nesta dinâmica apenas as três primeiras fases do MDCS, uma vez que elas estão orientadas à criação de estímulos que servem de base para engajar os indivíduos no processo de mudança comportamental (LUDDEN; HEKKERT, 2014). A etapa de atenuação gradual não foi observada, pois pressupõe que o novo comportamento já esteja assimilado pelo usuário. As informações no workshop foram coletadas por meio da técnica de observação participante. A partir da análise do processo de criação das equipes e dos jogos desenvolvidos, investigou-se de que maneira os grupos apropriaram-se do MDCS e como as fases do modelo foram traduzidas nos jogos. Das quatro equipes participantes, duas estabeleceram uma relação direta entre o jogo e o modelo, criando uma atividade para cada fase do MDCS. Os outros grupos propuseram dinâmicas somente para a fase de sensibilização. A Figura 2 demonstra como cada equipe utilizou o MDCS para compor os seus projetos.

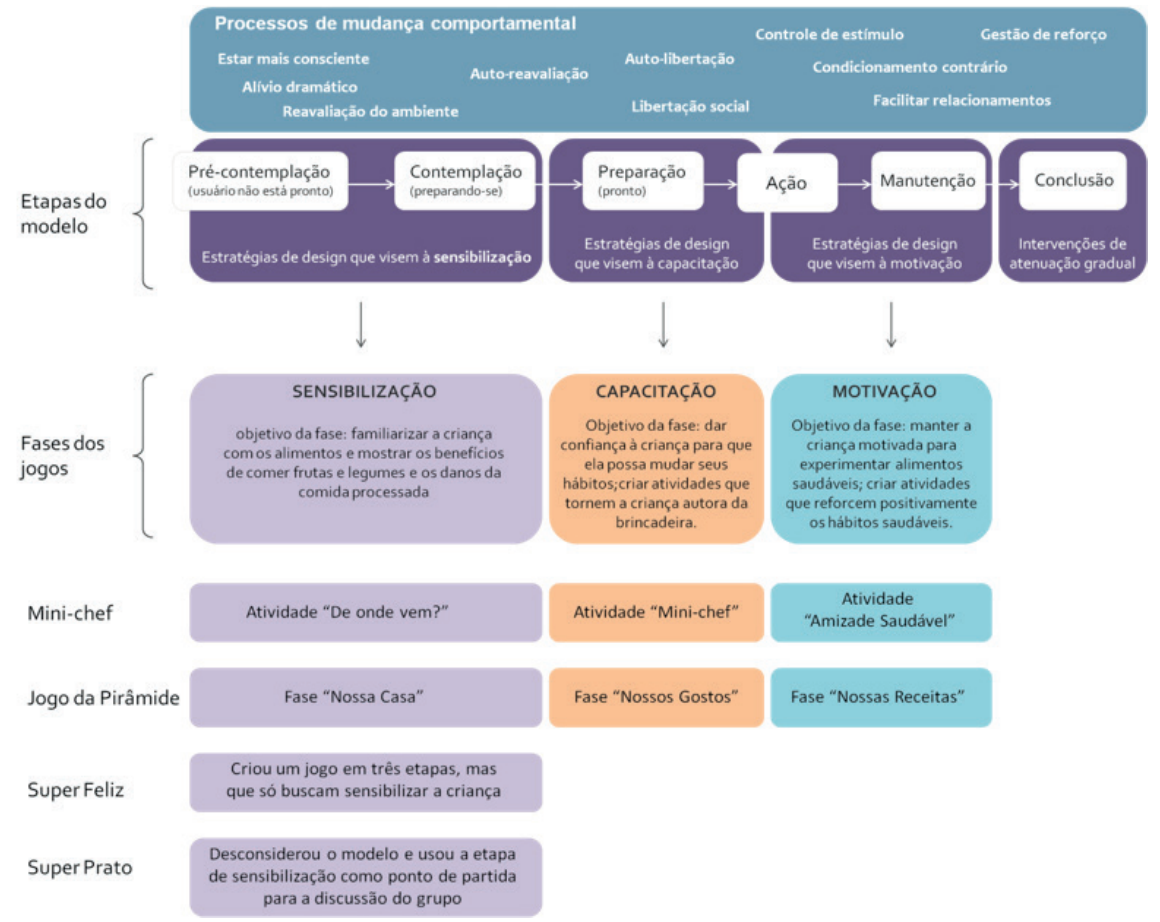

Figura 2: Aplicação do MDCS no workshop. Fonte: elaborada pela autora. 


\subsection{JOGO MINI-CHEF}

Este jogo propôs uma atividade de interação específica para cada fase do MDCS. A tarefa "De onde vem?" foi desenvolvida para atender à etapa de sensibilização do modelo, que sugere a criação de artefatos para aumentar a consciência do indivíduo sobre a importância dos hábitos saudáveis. Com base nesta compreensão, a equipe de design criou esta dinâmica que propõe que as crianças visitem os locais onde os alimentos saudáveis são produzidos, para que possam se familiarizar melhor com a comida nutritiva.

A atividade "Mini-chef" foi projetada para responder à etapa de capacitação do MDCS, que trata da criação de estímulos que proporcionem confiança para o indivíduo avançar no processo de mudança comportamental. Nesta tarefa do jogo, a criança deve escolher um ajudante especial e preparar uma nova receita com ingredientes saudáveis para compartilhar com a família. Através desta dinâmica, o grupo buscou atingir a criança que já está familiarizada com a alimentação saudável, mas que precisa de suporte para manter os hábitos saudáveis. Para tanto, a atividade foi projetada com o objetivo de estimular as habilidades da criança no preparo dos alimentos e fortalecer sua autoestima, mantendo-a motivada a interagir e experimentar alimentos saudáveis.

A fim de atender à etapa de motivação do MDCS, a equipe desenvolveu a atividade "Amizade Saudável". Esta fase do modelo diz respeito à criação de estímulos constantes que reforcem o comportamento saudável de maneira que ele seja aprendido pelo indivíduo e se torne um hábito. A tarefa do jogo propõe que a criança prepare uma refeição saudável e compartilhe com os amigos. Esta dinâmica tem o propósito de fortalecer o comportamento saudável por meio da afetividade da criança com amigos e da diversão proporcionada pela experiência. A análise do jogo Mini-chef aponta, neste sentido, a preocupação dada equipe de design em atender objetivamente a cada etapa do MDCS e dar a elas a mesma atenção na concepção do jogo, a fim de criar uma proposta mais consistente e estimulante à criança. Neste grupo, o modelo serviu como um condutor do processo de criação das ideias, na medida em que as usuárias e a designer recorreram constantemente a ele para ter subsídios à criação de ideias e avançar no desenvolvimento do jogo.

\subsection{JOGO DA PIRÂMIDE}

De forma semelhante ao grupo anterior, o Jogo da Pirâmide criou uma dinâmica de interação da criança para cada fase do MDCS. A primeira etapa do jogo

- Nossa Casa - incentiva a criança a conhecer novas frutas e legumes e a montar 
uma pirâmide alimentar com os alimentos saudáveis disponíveis em casa. No entendimento da equipe, esta dinâmica está associada à fase de sensibilização do modelo de design, pois auxilia a criança a conhecer melhor os benefícios da alimentação balanceada.

Para atender à fase de capacitação do modelo de design, o grupo criou a atividade "Nossos Gostos", que estimula a criatividade dos participantes ao propor a criação de combinações criativas com frutas e legumes no caderno de receitas. Associada à etapa de motivação do MDCS, o grupo desenvolveu a atividade "Nossas Receitas", que propõe que os pais e a criança testem as receitas criadas, associando os alimentos saudáveis à experiência positiva da convivência em família. Esta tarefa foi projetada com o objetivo de manter constante a motivação das crianças em experimentar alimentos nutritivos e tornar essa prática um hábito.

A análise mostrou que o grupo conseguiu delimitar com mais precisão as fases do jogo e os objetivos a serem atingidos por cada uma, estabelecendo, assim, uma relação clara com o modelo de design. Assim como a proposta anterior, o Jogo da Pirâmide revelou a preocupação da equipe em estabelecer um equilíbrio entre as três fases do MDCS e criar atividades que proporcionassem uma experiência envolvente e criativa da criança com a alimentação saudável.

Neste grupo, o MDCS também foi adotado como um balizador do processo de criação de ideias, na medida em que as usuárias e designers recorreram a ele constantemente para verificar se o jogo desenvolvido conseguia traduzir cada etapa do modelo. Além disso, o modelo ajudou a equipe a organizar os conceitos gerados de dentro de um jogo com dinâmicas interligadas que atendessem ao objetivo do workshop.

\subsection{JOGO SUPER FELIZ}

O jogo Super Feliz também foi estruturado em três fases para responder às etapas de sensibilização, capacitação e motivação do MDCS. A análise revelou, no entanto, que as atividades dão mais ênfase à sensibilização, concentrando-se principalmente na familiarização da criança com a comida saudável.

A primeira atividade do jogo Super Feliz consiste na apresentação de cartas com informações nutricionais de alimentos saudáveis e industrializados para a criança. Diante das diferentes opções de alimentos, ela deve escolher quais ela gostaria de inserir no tabuleiro que acompanha o jogo e alimentar o personagem. Com a ajuda dos pais, a criança avalia se as cartas selecionadas ilustram alimentos nutritivos ou não. O propósito da atividade, segundo as usuárias e a designer, é ensinar aos jogadores os benefícios da alimentação saudável e os 
danos da comida processada, oferecendo um suporte para que eles aprendam a fazer escolhas alimentares mais positivas.

A segunda dinâmica do jogo envolve a criação de um placar com o desempenho alimentar da criança na semana. À medida que ela prova novos alimentos saudáveis, a criança recebe maior pontuação através de ícones com um sorriso. Atitudes como a irritação nas refeições rendem um ícone triste no placar. Por meio desta dinâmica, o grupo buscou estimular a criança a superar o seu próprio desempenho e a experimentar novos sabores.

Por fim, a terceira atividade proposta no jogo Super Feliz sugere que a criança experimente um dos alimentos saudáveis contidos nas cartas que ela escolheu no início da dinâmica. O objetivo desta tarefa é estimular a familiarização da criança com novos alimentos e aumentar o seu repertório alimentar.

A partir da análise das etapas do jogo, é possível concluir que, embora seja constituído por três momentos, ele não respeita as características de cada etapa do processo de mudança de comportamento como estabelece o MDCS. Os estímulos desenvolvidos, neste caso, atendem somente ao princípio da sensibilização do modelo, que tem por objetivo aumentar o nível de consciência do indivíduo sobre a importância dos hábitos saudáveis e ensiná-lo a fazer escolhas mais positivas para o seu bem-estar.

A análise demonstra que, em comparação com as propostas anteriores, o grupo Super Feliz não compreendeu de forma clara as etapas do MDCS. Neste grupo, o modelo acabou servindo como um recurso secundário no desenvolvimento das propostas.

\subsection{JOGO SUPER PRATO}

O grupo Super Prato, diferentemente dos anteriores, não criou seu jogo com base nas fases do modelo de design. $O$ jogo consiste em um baralho de cartas com a ilustração de alimentos saudáveis e industrializados. As cartas possuem pontos que variam de 1 a 5 de acordo com o valor nutricional do alimento representado (frutas e verduras garantem cinco pontos aos jogadores, e os alimentos industrializados oferecem apenas um). O objetivo do jogo é conquistar mais pontos através das cartas selecionadas para compor um prato imaginário. O grupo entendeu que as premissas de cada fase do MDCS podiam estar de forma diluída nas atividades de interação, sem estarem demarcadas por estágios a serem cumpridos pelos jogadores. No entendimento da equipe, o jogo organizado nesta lógica poderia atingir crianças com diferentes padrões nutricionais, desde as que não são familiarizadas com a alimentação balanceada até aquelas 
que já assimilaram os hábitos nutricionais saudáveis.

Apenas a fase de sensibilização recebeu a atenção do grupo, de acordo com os diálogos revelados no processo de projeto. A primeira fase do MDCS serviu apenas como ponto de partida para a troca de ideias da equipe. Nas discussões subsequentes, o grupo desconsiderou as características e objetivos das fases de capacitação e de motivação. Corrobora com este resultado o fato das dinâmicas do jogo concentrarem-se somente na difusão das informações nutricionais dos alimentos, a fim de que a criança aprenda os benefícios de cada um e realize escolhas mais saudáveis.

\section{CONSIDERAÇÕES FINAIS}

A avaliação do processo criativo das equipes demonstrou que, nas equipes do workshop que utilizaram o MDCS como suporte para o projeto (Mini-chef e Jogo da Pirâmide), o modelo auxiliou na condução do processo criativo e no desenvolvimento de jogos que atenderam de forma mais precisa e consistente ao briefing. Com o apoio do MDCS, estas equipes conseguiram projetar estímulos específicos para cada fase da mudança comportamental da criança, gerando atividades interativas através das quais ela pudesse aprender gradualmente novos padrões alimentares e manter-se motivada nesse processo.

Nas equipes do Jogo da Pirâmide e Mini-chef, o MDCS revelou-se como um instrumento balizador do processo de projeto, servindo de apoio à ideação e à tomada de decisões mais consistentes para atender ao objetivo do workshop. Além disso, nestes grupos, o MDCS auxiliou as usuárias e designers a validarem se as atividades propostas atendiam às diretrizes do briefing, comparando-as às fases do modelo.

Embora a equipe responsável pelo jogo Super Feliz tenha recorrido ao modelo como um apoio para a criação das atividades de interação, a análise revelou que as usuárias e designers não compreenderam as diferenças entre as etapas de sensibilização, capacitação e motivação. A exceção foi o jogo Super Prato, cuja equipe não se apoiou no modelo para auxiliar a condução do processo, utilizando-o somente como um elemento de discussão inicial das ideias.

As propostas dos jogos demonstraram, nesse sentido, que o modelo, quando foi utilizado e compreendido pelos atores do projeto, operou como um fio condutor do processo de concepção das ideias, ajudando-os a organizar os jogos dentro de uma lógica encadeada de etapas. Neste sentido, foi possível observar que o MDCS ajudou a potencializar a dinâmica de projeto orientado à mudança comportamental, pois ofereceu parâmetros que ajudaram a balizar o processo de ideação e 
a direcioná-lo para responder ao problema de design de forma mais precisa.

O uso do MDCS contribuiu também para a criação de estímulos que reforçam com mais frequência o comportamento alimentar saudável da criança. Esta característica foi observada de forma mais explícita no Jogo da Pirâmide e no jogo Mini-chef, justamente aqueles que foram projetados dentro da lógica do modeIo. O MDCS parte do princípio de que a aprendizagem de novos hábitos ocorre em uma sequência de etapas pelas quais o indivíduo deve percorrer em um período de tempo. Para que possa incentivar o usuário neste processo, os designers devem criar estímulos específicos para cada uma destas fases. Por esta razão, as equipes que desenvolveram seus jogos com base no MDCS acabaram gerando estímulos que operam de forma sucessiva sobre os hábitos da criança, aumentando sua propriedade de fortalecer o comportamento saudável.

Ao estabelecer uma conexão entre as fases do projeto e os estágios da aprendizagem de novos comportamentos, o MDCS propõe uma lógica inversa da atividade projetual: ao invés da criação de soluções para o modo como o usuário gostaria de agir, é mais efetivo projetar artefatos que atendam às necessidades específicas do indivíduo em cada etapa da mudança comportamental, motivando-o gradualmente a avançar neste processo. O MDCS, neste sentido, traz uma nova perspectiva para a criação de soluções para o comportamento saudável, na medida em que respeita a trajetória e as motivações do usuário no processo de mudança, assumindo, assim, uma abordagem menos impositiva sobre as ações do indivíduo.

Através do uso do MDCS como suporte para a criação de jogos focados na educação nutricional infantil, é possível concluir que o modelo impacta o modo de projetar artefatos para o bem-estar na perspectiva do design para mudança de comportamento. Ao apontar diretrizes que orientam a criação de estímulos específicos para cada etapa da mudança comportamental, o MDCS torna o processo de projeto mais objetivo e eficiente. Este modelo coloca-se, portanto, como um instrumento de design que respeita as propriedades de cada fase da aprendizagem de novos comportamentos e potencializa a criação de soluções para incentivar hábitos saudáveis. Para o desenvolvimento de futuros projetos, sugere-se a realização das etapas subsequentes do projeto, de prototipação e avaliação com as crianças, de maneira que se possa analisar a eficiência dos jogos no estímulo de comportamentos alimentares saudáveis.

\section{REFERÊNCIAS}

BHAMRA, T. A.; LILLEY, D.; TANG, T. Sustainable Use: Changing Consumer 
Behaviour Through Product Design. In: CIPOLLA, C.; PERUCCIO, P. (Eds.). Changing the Change: Design, Visions, Proposals and Tools: Proceedings. Italy: Torino, 2008. p. 1-13. Disponível em: <http://www.allemandi.com/university/ ctc.pdf>. Acesso em: 31 jan. 2017.

BUCHANAN, R. Design Research and the New Learning. Design Issues, Cambridge (MA), v. 17, n. 4, p. 3-23, May. 2001.

LILLEY, D. Design for Sustainable Behaviour: Strategies and Perceptions. Design Studies, Oxford, v. 30, n. 6, p. 704-720, 2009.

LOCKTON, D.; HARRISON, D.; STANTON, N. The Design with Intent Method: a Design Tool for Influencing User Behavior. Applied Ergonomics, [s.I.], v. 41, n. 3, p. 382-392, may. 2010.

LUDDEN, G.; HEKKERT, P. Design for Healthy Behavior Design Interventions and Stages of Change. In: SALAMANCA, J., DESMET, P., BURBANO, A., LUDDEN, G., MAYA, J. (Eds.). Proceedings of the Colors of Care: The 9th International Conference on Design \& Emotion. Ediciones Uniandes, Bogotá, 2014. p. 482-488. MURILLO, A. et al. Modeling Eating Behaviors: the Role of Environment and Positive Food Association Learning via a Ratatouille Effect. Mathematical Biosciences and Engineering, [s.I.], v. 13, n. 4, p. 841-855, 2016.

NIEDDERER, K. et al. Creating Sustainable Innovation through Design for Behaviour Change: Full Project Report. Leicestershire, UK: Project Partners \& AHRC, 2014.

PROCHASKA, J. O.; DICLEMENTE, C. C.; NORCROSS, J. C. In Search of How People Change. American Psychologist, [s.I.], 47, n. 9, p. 1102-1114, 1992.

TROMP, N. Social Design: How Products and Services Can Help us Act in Ways that Benefit Society. 224 f. 2013. (Ph.D.thesis) - Industrial Design Engineering, Delft University of Technology, Netherlands, 2013.

Filipe Campelo Xavier da Costa é professor e Pesquisador do Programa de Pós-Graduação em Design da Universidade do Vale do Rio dos Sinos. Doutorado em Administração pela Universidade de São Paulo, Mestrado e Graduação em Administração pela Universidade Federal do Rio Grande do Sul. Os temas de in- 
teresse de pesquisa envolvem Design para Experiência e para Emoção, Design para mudança de comportamento e processos de consumo.

Juliana Gonçalves Mota possui graduação em Comunicação Social - Habilitação em Publicidade e Propaganda, pela Universidade Federal do Rio Grande do Sul (2007) e especialização em Economia da Cultura pela mesma instituição (2011). É mestre em Design Estratégico pela Universidade do Vale do Rio dos Sinos (2017). Atua como gestora de projetos na Secretaria de Comunicação Social da Universidade Federal do Rio Grande do Sul. Tem experiência na área de Artes e Comunicação, com ênfase em Produção Cultural. Pesquisa temas na área do design para mudança de comportamento e experiência do usuário. 